\title{
STATUS OF PI OF THE SKY TELESCOPES IN SPAIN AND CHILE
}

T. Batsch ${ }^{1}$, H. Czyrkowski ${ }^{2}$, M. Cwiok ${ }^{2}$, R. Dabrowski ${ }^{2}$, G. Kasprowicz ${ }^{3}$, A. Majcher ${ }^{1}$, A. Majczyna ${ }^{1}$, K. Malek ${ }^{4,5}$, L. Mankiewicz $^{4}$, K. Nawrocki $^{1}$, R. Opiela ${ }^{4}$, L.W. Piotrowski ${ }^{2}$, M. Siudek ${ }^{4}$, M. Sokolowski ${ }^{1}$, R. Wawrzaszek ${ }^{6}$, G. Wrochna ${ }^{1}$, M. Zaremba ${ }^{2}$ and A.F. Żarnecki ${ }^{2}$

\begin{abstract}
Pi of the Sky is a system of wide field-of-view robotic telescopes which search for short timescale astrophysical phenomena, especially for prompt optical GRB emission. The system is designed for autonomous operation, follows the predefined observing strategy and adopts it to the actual conditions. We describe the current status of telescopes located in Chile and Spain and prospects for future development.
\end{abstract}

\section{Introduction}

The "Pi of the Sky" (Burd et al. 2005) is a system of wide field of view robotic telescope designed for efficient search for astrophysical phenomena varying on scales from seconds to months, especially for prompt optical counterparts of Gamma Ray Bursts (GRBs). The design of the apparatus allows to monitor a large fraction of the sky with a range of $12^{\mathrm{m}}-13^{\mathrm{m}}$ and time resolution of the order of $1-10$ seconds. In order to ensure that all project requirements are met with full control over the detector design and construction, "Pi of the Sky" detectors are equipped with custom designed CCD cameras, built by project members. Each camera is equipped with Canon lenses $\mathrm{f}=85 \mathrm{~mm}, \mathrm{f} / \mathrm{d}=1.2$ and covers $20^{\circ} \times 20^{\circ}$ of the sky.

\footnotetext{
1 National Centre for Nuclear Research, Hoza 69, 00-681 Warsaw, Poland

2 Faculty of Physics, University of Warsaw Hoza 69, 00-681 Warsaw, Poland

3 Institute of Electronic Systems, Warsaw University of Technology, Nowowiejska 15/19, 00-665 Warsaw, Poland

4 Centre for Theoretical Physics of the Polish Academy of Sciences, Al. Lotnikow 32/46, 02-668 Warsaw, Poland

${ }^{5}$ Institute for Advanced Research, Nagoya University, Furo-cho, Chikusa-ku, 464-8601 Nagoya, Japan

6 Space Research Center of the Polish Academy of Sciences, Bartycka 18A, 00-716 Warsaw, Poland
} 
The full "Pi of the Sky" system, which is under construction now will be capable of continuous observation of about 1.5 steradians, which is roughly corresponding to the field of view of the BAT instrument on board the Swift satellite (Gehrels et al. 2004). In October 2010 the first unit of the new Pi of the Sky detector system was successfully installed in the INTA El Arenosillo Test Centre in Spain. The first site of the ultimate system should be fully operational next year, in the same location.

\section{Observations}

\subsection{Observational strategy continuous observation of large part of the sky}

Observations of optical counterparts of GRBs during or even before the gammaray emission are crucial for understanding the nature of GRBs. The standard approach, which relies on waiting for an alert distributed by the GCN network (The Gamma Ray Burst Coordinates Network; Barthelmy et al. 1998) and subsequently moving the telescopes to the target as fast as possible, does not allow us to detect an outburst at the moment of or before the GRB explosion. Thus, the "Pi of the Sky" strategy is based on continuous observation of a large fraction of the sky which increases the chances that a GRB will occur in the observed area. Following the field of view of the Swift satellite, with the full "Pi of the Sky" system, will allow to eliminate a delay of the observation due to telescope re-pointing to the coordinates from GCN. Dead time, which arises from the decision process and signal propagation from the satellite to the GCN and from the GCN to the ground instruments is eliminated as well.

The search for GRB requires very fast data processing and identification of events in real-time. On the other hand, the search for transients and the analysis of variable star are based on precise photometry which requires detailed image analysis. To fulfill both requirements we developed two different sets of algorithms: for on-line and off-line data processing. Off-line analysis is to identify all objects in an image, and to add their measurements to the database. The on-line algorithm searches for flashes in real-time by comparing a new image with the stack of recently taken frames. Any observed difference is considered as possible candidate event. All events are processed through a multilevel triggering system similar to those known from high-energy physics experiments.

The observations of the famous "naked-eye" GRB 080318B (Racusin et al. 2008) have confirmed the usefulness of "Pi of the Sky" strategy Wide-field telescopes performing continuous observations of large part of the sky are capable of detecting GRBs at the moment or even before explosion. GRB 080318B was recognized by the "Pi of the Sky" self-triggering system independently from the alert received from the GCN.

\subsection{The "Pi of the Sky" prototype}

Before constructing the final version, tests of hardware and software were performed with a prototype consisting of 2 custom-designed cameras placed on an equatorial mount. The detector is fully autonomous and operates without any 
human supervision, although remote control via Internet is possible as well. Cameras work in coincidence and observe the same field of view with a time resolution of $10 \mathrm{~s}$. The limiting magnitude for a single frame is $12^{\mathrm{m}}$ and rises to $13.5^{\mathrm{m}}$ for a frame stacked from 20 exposures. Till 2009 all observations were made in white light and no filter was used, except for an IR-cut filter in order to minimize the sky background. Since May 2009 we have had a Bessel-Johnson R-band filter installed on one of the cameras in order to facilitate absolute calibration of the measurements. The prototype had been working at Las Campanas Observatory in Chile since June 2004 till the end of 2009. In March 2011 the detector was moved to a new site in San Pedro de Atacama, approximately $750 \mathrm{~km}$ north from LCO (still in Chile) and about 2400 meters above sea level.

During the period 2006-2009 the prototype has gathered over 2 billion measurements for almost 17 million objects. All measurements acquired by "Pi of the Sky" are publicly accessible through a user-friendly web interface on the Pi of the Sky Home Page. Effort on improving data quality is still ongoing. We have developed a system of dedicated filters to remove measurements from star light curve measurements, which could be affected by different factors due to detector imperfections or weather conditions. The measurement quality can be improved by an approximate color calibration algorithm based on the spectral type of reference stars and an uncertainty of the order of $0.013^{\mathrm{m}}$ can be obtained.

\section{New detector unit in Spain}

The final detector consists of 4 custom-designed CCD cameras, which are improved versions of the cameras developed for the prototype. The cameras can operate in two operational modes thanks to specially designed equatorial mount. The mechanism for moving cameras against the main instrument axis enablea to point all cameras at the same object (common-target mode, DEEP common field of view $20^{\circ} \times 20^{\circ}$ ) or cover adjacent fields (side- by-side, WIDE, total coverage $40^{\circ} \times 40^{\circ}$ ) Due to numerous improvements, the new design of the telescope mount provides much better pointing accuracy and a shorter reaction time than the prototype. New detector unit has been successfully operated in the INTA El Arenosillo test centre in Mazagón near Huelva, Spain, on the coast of the Atlantic Ocean from October 2010. The ultimate system, consisting of 4 such units, will be operational in 2013.

We are very grateful to G. Pojmanski for access to the ASAS dome and sharing his experience with us. We would like to thank the staff of the Las Campanas Observatory San Pedro de Atacama Observatory and the INTA El Arenosillo test centre in Mazagón near Huelva for their help during the installation and maintenance of our detector This work has been financed by the Polish Ministry of Science and Higher Education in 2009-2012 as a research project.

\section{References}

Barthelmy, S.D., Butterworth, P., Cline, T.L., et al., 1998, AIPC, 428, 99

Burd, A., Cwiok, M., Czyrkowski, H., et al., 2005, New Astron., 10, 409

Gehrels, N., Chincarini, G., Giommi, P., et al., 2004, ApJ, 611, 1005

Racusin, J.L., Karpov, S.V., Sokolowski, M., et al., 2008, Nature, 455, 183 
